\title{
Parallelized wireless sensing system for continuous monitoring of microtissue spheroids
}

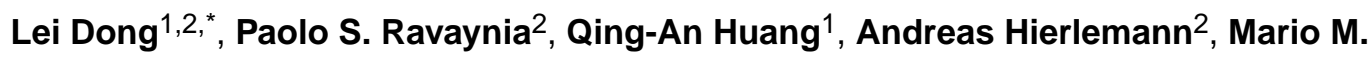 \\ Modena $^{2, *}$
}

${ }^{1}$ Southeast University, Key Laboratory of MEMS of the Ministry of Education, Nanjing 210096, China ${ }^{2}$ ETH Zürich, Department of Biosystems Science and Engineering, Basel 4058, Switzerland

\section{Abstract}

Currently, the use of electrical readout methods for the investigation of microtissue spheroids in combination with lab automation tools is hindered by the cable connections that are required to interrogate the on-chip-integrated electrodes. To overcome this limitation, we developed a wireless sensor scheme, which can detect the size variation of microtissues during long-term culturing and drug exposure assays. The sensor system includes an interrogation board, which is composed by an inductor-capacitor (LC) readout circuit, and the tissue culture platform with integrated split-ring sensors. The magnetic coupling between the LC circuit and the sensors enables the interrogation of the on-chip sensors without any wire connection to the culture platform. By optimizing the sensor dimensions and the LC resonance frequencies, we were able to avoid cross talk between neighboring sensors. We integrated 12 tissue compartments on a standard microscopy slide with a sensor-to-sensor pitch of $9 \mathrm{~mm}$, which is in accordance with standard 96 well-plate dimensions. As proof-of-concept experiment for the developed system, we monitored continuously and during more than four days the growth inhibition of colon-cancer microtissue spheroids that had been exposed to different concentration of doxorubicin, a chemotherapeutic compound. The stability of the measurements during long-term culturing and the compatibility of the sensor scheme with standard lab equipment offers great potential for automated electrical microtissue spheroid characterization.

\section{Keywords}

biosensors; inductor-capacitor; microtissue spheroids; split ring; wireless sensing system

Microtissue spheroids are an emerging in vitro tissue model that has been used in fundamental biology and for the identification and selection of novel therapeutics. ${ }^{1-3}$ Compared to two-dimensional cell layers, the three-dimensional structure of a microtissue

\footnotetext{
*Corresponding Authors: Lei Dong, dl@ seu.edu.cn. Tel: +86 258379 2632. Fax: +86 258379 2939; Mario M. Modena, mario.modena@bsse.ethz.ch. Tel: +41 613873344.

Notes

The authors declare no competing financial interest.
} 
spheroid enables to recapitulate cell-to-cell and cell-to-extracellular matrix interactions and to better mimic tissue organization and functionality. ${ }^{4,5}$ Owing to their self-assembly tissue formation, their high biological relevance and their ease of handling, spheroids are becoming increasingly popular models for testing drug compounds. ${ }^{6,7}$ Therefore, different characterization methods have been proposed that feature high throughput and automated operation, both of which are required for use in screening of large compound libraries. ${ }^{8,9}$

Electrical impedance spectroscopy (EIS) is a label-free characterization method that has been used for the investigation of different biological samples, ranging from single cells and 2D cell monolayers to multicellular constructs and organisms. ${ }^{10-14}$ EIS can be used to measure the dielectric properties of a sample under investigation by applying an AC electric field at different frequencies across the sample through a set of electrodes. ${ }^{15}$ Tissue apoptosis upon drug exposure, for example, could be detected by analyzing the variations in the impedance spectrum of a spheroid in a small microcavity featuring electrodes on the sidewalls. ${ }^{16}$ The long-term growth characterization of multiple microtissue spheroids was obtained by making the tissues roll over a pair of co-planar electrodes and by measuring the increase in impedance between the electrodes upon passage of the spheroid. ${ }^{17} \mathrm{~A}$ similar approach was used to record the beating of cardiac microtissues, cultured in a hanging-drop network, by using integrated electrodes in the fluidic support structure. ${ }^{18}$ However, although electrical measurements offer a high degree of parallelization, the use of cables and electrical connections that provide the AC signals to the sensing electrodes strongly limits the use of EIS-based platforms with automated liquid handling systems and robotic arms in high-throughput applications.

Wireless sensors based on inductor-capacitor (LC) radiating circuits enable to interrogate integrated sensors when physical cable routing is either not possible or would interfere with the experimental procedure. ${ }^{19} \mathrm{LC}$ wireless sensors have been widely employed for a large range of applications, from bacteria detection on teeth ${ }^{20}$ to monitoring of internal physiological parameters in healthcare application ${ }^{21}$ or to the measurement of local $\mathrm{pH}$ values using nanoparticle-functionalized hydrogels. ${ }^{22}$ This type of sensors exploits the capability of an LC circuit to emit electromagnetic waves upon application of an AC current. The radiated power reaches a maximum when the frequency of the applied signal matches a resonance frequency of the LC circuit: Variations in the LC sensor environment are reflected in variations of resonance frequency and/or amplitude of the radiated signal.

Here, we introduce a wireless impedance-based platform for the characterization of cancer microtissues. The platform is composed of a LC readout board and a tissue-culture chip with culture wells that feature integrated split-ring (SR) resonators as sensing units. The SR is a particular type of electrical resonator, realized as a metal trace of square geometry, which features a gap in one of the 4 bases. The SR sensors were patterned on a glass substrate, which was then bonded to a polydimethylsiloxane (PDMS) chip. The PDMS chip was structured as an array of microwells of conical shape for microtissue culturing. The SR gaps were aligned with the well bases and in contact with the cell-culture medium. In close vicinity to an emitting LC circuit, the SR was excited through magnetic coupling, and a set of currents was induced on the sensor trace, which behaved as an LC circuit. ${ }^{23}$ Placing a microtissue spheroid in the culture well caused a displacement of the conductive medium in 
the SR gap with less conductive cell or tissue material, which resulted in an increase of the SR impedance. Finally, this impedance variation caused an increase in the total impedance perceived by the interrogating LC circuit due to the magnetic coupling between the LC readout circuit and the SR sensor. As the impedance variation is directly proportional to the volume of the medium that has been displaced by the spheroid, any change in microtissue size could be detected wirelessly by monitoring the impedance variations in the readout LC circuit. To validate the wireless sensing system in monitoring microtissue growth and to demonstrate parallelizability, we developed a platform featuring 12 culture wells, probed by 12 separate readout channels, which were centered at different resonance frequencies. We tested our integrated tissue-culture system by continuously measuring the size variation of cancer microtissues that had been exposed to different concentrations of doxorubicin, a known chemotherapeutic agent, for over 90 hours.

\section{Experimental Section}

\section{SR Sensor Chip}

The SR sensor board included a glass substrate $\left(75 \times 25 \times 0.5 \mathrm{~mm}^{3}\right)$ with SR-patterned metal traces, which was bonded to a PDMS microfluidic structure. The 200-nm-thick platinum metal traces were patterned using a standard lift-off process (Figure S1). Each chip was equipped with $12 \mathrm{SR}$ sensors at $5 \mathrm{~mm}$ spacing between them. The split ring featured an outer diameter of $4 \mathrm{~mm}$, a trace width of $1 \mathrm{~mm}$ and an SR gap of $500 \mu \mathrm{m}$.

The PDMS microfluidic structure consisted of an inverted-pyramid well structure above each SR gap. The top surface of each pyramid was shaped as a square of $5 \mathrm{~mm}$ base length, while the open-bottom face featured a square shape with $600 \mu \mathrm{m}$ base length. The small bottom opening ensured the correct placement of the spheroid on top of the SR gap. The height of the PDMS chip was $5.5 \mathrm{~mm}$, and each well could host up to $50 \mu \mathrm{L}$ of cell culture medium. The culture wells were coated with Biolipidure 206 (NOF America Corporation, White Plains, NY, USA) to prevent cell attachment and to increase the hydrophilicity of the surface.

\section{Sample Preparation and Chip Loading}

Human colorectal cancer cells, HCT-116, were purchased from ATCC (ATCC - LGC Standards GmbH, Wesel, Germany) and maintained in RPMI-1640 medium (Fisher Scientific France, Illkirch Cedex, France), supplemented with 10\% FBS (Sigma Aldrich Chemie GmbH, Buchs, Switzerland) and 1\% penicillin/streptomycin (Fisher Scientific France) in T25 flasks (Greiner Bio-One International GmbH, Frickenhausen, Germany). Cells were passaged every 2-3 days ( $80 \%$ confluency) at a 1:5 ratio. Microtissue spheroids were prepared prior to the experiments by loading $100 \mu \mathrm{L}$ of HCT-116 cell suspension (5000 cells $/ \mathrm{mL}$ ) per well of an ultra low attachment U-bottom plate (ThermoFisher Scientific, Schwerte, Germany). After 72 hours of incubation at $37^{\circ} \mathrm{C}, 5 \% \mathrm{CO}_{2}$ and $95 \%$ relative humidity, microtissue spheroids of $\sim 300 \mu \mathrm{m}$ diameter were formed.

Before use, the sensor chip and the readout platform were sterilized by overnight UV exposure. First, $50 \mu \mathrm{L}$ of culture medium were introduced into each well and, subsequently, 
pre-formed spheroids were transferred into each well. For the drug assay, doxorubicin (Sigma-Aldrich Chemie $\mathrm{GmbH}$ ) was added to the culture medium to obtain final concentrations of $0.1 \mu \mathrm{M}, 1 \mu \mathrm{M}$ and $10 \mu \mathrm{M}$. The media with the drug compound were then loaded in the chip wells before starting the assay. As control, microtissue spheroids were also cultured in low-attachment U-bottom plates (see Supporting Information).

\section{Experimental Setup}

A lock-in amplifier (UHF, Zurich Instruments, Zurich, Switzerland) was used to interrogate the readout board. The input and output ports of the lock-in amplifier were connected to the readout platform via a T-connector. The lock-in amplifier was used to generate an AC signal that was swept from $100 \mathrm{MHz}$ to $380 \mathrm{MHz}$ (step size $0.07 \mathrm{MHz}$ ) with $\mathrm{V}_{\mathrm{pk}}$ of $750 \mathrm{mV}$, which was fed to the readout platform. The signal acquisition of each frequency sweep took $\sim 30 \mathrm{~s}$.

The sensor chip and readout platform were aligned and held in position using a standard microscopy-slide holder (Microfluidic ChipShop GmbH, Jena, Germany). Each holder could hold up to 4 chips in parallel. During long-term measurements, two SR chips were loaded with spheroids for testing, while we placed chips filled with sterile water in the remaining two positions to reduce evaporation. The whole assembly was then placed in the incubator.

First, baseline signals of the 12 resonance peaks were acquired by loading only cell-culture medium in the culture wells. After loading the microtissue spheroids, the voltage amplitude at the resonance frequencies was recorded, and the voltage difference at resonance was used to extract information on the spheroid size.

\section{Microtissue Size Measurement}

The microtissue size was characterized by optical microscopy at day 0 (when the culture medium was first introduced into the wells), day 2 (when the culture medium was exchanged) and day 4 (end of the assay). Image acquisition and size determination was performed using a plate scanner (Cell3iMager Neo cc-3000, Screen Holdings Co. Ltd., Kyoto, Japan).

\section{Results and Discussion}

\section{Electrical Equivalent Model}

We first developed an electrical equivalent model of the sensing system to investigate the coupling between the readout circuit and the SR sensor and the contribution of the microtissue in the SR gap to the measured impedance at the input port of the readout circuit (Figure 1).

The readout circuit is realized as a parallel RLC circuit, where L1 is the inductance of the readout coil, $\mathrm{C}_{1}$ and $\mathrm{R}_{1}$ are the parallel capacitance and the series parasitic resistance of the inductor coil, respectively. The SR equivalent model comprises the inductance $L_{S I}$ and resistance $R_{S I}$ of the metal trace and the capacitance $C_{S I}$ of the sensing area in parallel to the microtissue-induced impedance $Z_{m I}$. $C_{S}$ depends on the relative permittivity of both the culture medium and the microtissue, which are denoted $\boldsymbol{\varepsilon}_{m e}$ and $\boldsymbol{\varepsilon}_{m i}$ in the inset in Fig. 1a. For frequencies above $10 \mathrm{MHz}$, the equivalent permittivity $\varepsilon_{m i}$ converges to $\varepsilon_{m e},{ }^{24}$ and the 
equivalent permittivity of $C_{S}$ can be approximated by the medium permittivity in the presence or absence of a microtissue in the SR gap. Therefore, the gap capacitance $C_{S I}$ can be treated as constant for high-frequency excitation. The impedance $Z_{m 1}$ of the sensing area arises from the resistance of the culture medium $R_{m e}$ and from the capacitive and resistive contributions of the microtissue, which are represented by $C_{m i}$ and $R_{m i}$ in the blue inset of Fig. 1a. As the capacitance $C_{m i}$ can be considered constant at high frequencies, ${ }^{24}$ variations in $Z_{m 1}$ are mostly dependent on variations of the medium resistance caused by medium evaporation or by microtissue size variations, both of which affect the effective volume of culture medium in the SR gap.

When the excitation frequency corresponds to a resonance frequency of the readout coil, the equivalent input impedance $Z_{\text {in } 1}$ reaches a maximum and can be written as

$$
Z_{i n 1}=\frac{L_{1}}{\left(R_{1}+Z_{r s 1}\right) C_{1}}=\frac{L_{1}}{\left(R_{1}+A_{1}\left(B_{1}+\frac{1}{Z_{m 1}}\right)\right) C_{1}},
$$

where $Z_{I S} 1$ is the reflected impedance of $Z_{S}$. The reflected impedance represents the equivalent impedance of the SR sensor perceived at the readout coil due to the inductive coupling between the sensor and the readout coil (numerical simulations and more details on the electrical equivalent model are reported in the Supporting Information).

The voltage, detected at the input end of the measurement device, can be expressed as

$$
V_{r}=\frac{Z_{0}}{Z_{\text {in } 1}+Z_{0}} \cdot U
$$

where $Z_{O}$ is the impedance of the measurement device and $U$ is the output voltage. Since the input impedance reaches a maximum at the resonance frequency $\omega_{r}$, the detected voltage then reaches a minimum, which generates a dip in the voltage amplitude upon performing a frequency-sweep analysis (shown in Figure 1b). The voltage amplitude $V_{r}$ at resonance is inversely proportional to the input impedance $Z_{\text {in } 1}$ (Eq. 2). As microtissue spheroids feature a much lower conductivity in comparison to the surrounding cell-culture medium, ${ }^{25,26}$ the presence of a microtissue in the SR gap increases the impedance $Z_{m 1}$ and, therefore, $Z_{i n 1}$, which ultimately results in a reduction of $V_{r}$ (Figure 1b). This variation of the signal amplitude is directly proportional to the spheroid size, with larger spheroids inducing a larger decrease of the signal amplitude at resonance. The resonance frequency, however, remains unaffected (more details in the Supporting Information).

\section{Split-Ring Sensor Characterization}

Three SR sensors of different outer radius $r_{S o}$ and trace width $W$ were fabricated to study the sensitivity of the sensor as a function of SR size. The sensors are defined as: $S 1, r_{S O}=5 \mathrm{~mm}$ and $w=1 \mathrm{~mm} ; S 2, r_{S O}=2 \mathrm{~mm}$ and $w=1 \mathrm{~mm} ; S 3, r_{S O}=2 \mathrm{~mm}$ and $w=0.5 \mathrm{~mm}$. Each sensor was interrogated with a corresponding readout coil of the same size (i.e., for $S 1$, the outer radius of the readout coil was $5 \mathrm{~mm}$ and the inner radius was $4 \mathrm{~mm}$ ). A PDMS well structure was bonded on the top surface of the sensors for hosting the cell-culture medium and for ensuring that the spheroid was placed in the SR gap (Figure S3). We loaded a 500- $\mu$ - 
diameter multicellular spheroid of human colorectal tumor cells (HCT-116) into each well of the SR sensors, and we recorded the voltage change $\Delta V$ at resonance frequency induced by the presence of the spheroid (Figure 2a). Sensor $S 1$ yielded a much larger $\Delta V$ due to its larger dimensions, which entailed a strong magnetic coupling. For the smaller sensors, the larger trace width of $S 2$ resulted in a stronger induction, which then yielded a stronger signal variation (as expected from Eq. 1). We further investigated the effect of the dielectric material between the sensor and the readout coil on the signal amplitude. The SR sensors and the readout coils were coaxially aligned, and different materials, namely air, acrylic glass and glass, were placed in between the two components (Figure 2a). Owing to their dielectric properties, no significant differences in signal amplitude were recorded for the three different materials.

We then characterized how the distance between the SR and the readout coil affected the signal amplitude. $S 2$ was selected due to its small footprint and larger signal with respect to $S 3$. The distance of the SR sensor from the readout coil was varied in both, axial (vertical) and perpendicular (horizontal) directions. As for the previous characterization, we loaded a 500- $\mu \mathrm{m}$-diameter HCT-116 spheroid under standard culturing conditions in the SR gap and measured the $\Delta V$ variation (Figure $2 \mathrm{~b}$ ). As can be expected from the dependence of the coupling efficiency on the distance between the SR and the interrogating coil (Eq. S3), $\Delta V$ exhibited a large decrease upon increasing the spacing between the two components. For a distance of $2 \mathrm{~mm}$ in the axial (vertical) direction, the signal amplitude decreased by more than $75 \%$, which indicates that the maximum working distance roughly corresponds to the coil geometric dimensions. Signal characterization in the presence of a horizontal offset $d_{h}$ was also carried out to characterize the minimum distance between SRs that is required to avoid crosstalk between sensing units. The offset $d_{h}$ is defined as the horizontal distance between the center of the readout coil and that of the SR sensor. The test was performed with the sensor $S 2$ in contact with the readout board $(d=0 \mathrm{~mm})$ and displacing the SR sensor in the direction parallel to the SR bases without gap. With increasing $d_{h}$, the overlap area of the sensor and the readout coil decreased, which caused a corresponding reduction in inductance on the SR. For $d_{h}=1 \mathrm{~mm}$, only two sides of the sensor partially overlapped with the corresponding sides of the readout coil, while the two sides perpendicular to the moving direction did no more overlap with the corresponding sides of the readout coil, but were still interacting with the radiated field. For $d_{h}=2 \mathrm{~mm}$, the sensor was almost outside of the reach of the magnetic field of the readout coil, and the signal decreased to $20 \%$ of the maximum signal. This result demonstrates that interactions between neighboring sensing units are negligible, if the SRs are arranged at a distance larger than the coil radius. Hence, multiple sensing units can be integrated in a single readout platform for increased throughput without the risk of potential signal disturbances through neighboring units.

\section{Parallelized Platform}

We subsequently designed a platform for the analysis of multiple microtissue spheroids in parallel. The platform was composed of two parts: a readout board, which contained the interrogating coils, and a tissue culture chip, which featured the SRs integrated in the base of the culture wells (Figure 3). The platform dimensions were $75 \times 25 \mathrm{~mm}^{2}$, to be compatible 
with standard microscopy slide holders, and the tissue wells were arranged at a 9-mm pitch, in accordance with standard 96-well plate formats.

The readout board was realized as a printed circuit board (PCB) with 12 planar coils of 2 mm outer radius $r_{r o}$, which formed a 2 × 6 array on the top side of the PCB (Figure $3 \mathrm{a}$ and Figure S4). The spacing $s$ between adjacent coils was $5 \mathrm{~mm}$. The width of the coil trace was $100 \mu \mathrm{m}$, the spacing between adjacent traces was $75 \mu \mathrm{m}$, and the inductance of each coil was adjusted by varying the number of windings of the coil. Each readout coil was connected in parallel to a distributed capacitor at the bottom side of the PCB board to tune and separate the resonance frequencies of the different resonators. The details of the coils, of the distributed capacitors and of the corresponding resonant frequencies are given in Table S1. The microtissue culture chip consisted of a glass substrate with platinum traces for the realization of the SRs $\left(r_{S O}=2 \mathrm{~mm}, w=1 \mathrm{~mm}\right)$, which then was bonded to a PDMS structure hosting the culture wells (Figure S1b).

The separation of the resonance frequencies allowed to interrogate the 12 culture wells independently by simply recording the response of the board to a frequency sweep from 100 $\mathrm{MHz}$ to $370 \mathrm{MHz}$ (Figure 3b). The presence of a microtissue in the SR gap only affected the peak amplitude, while the resonance frequency depended on the LC characteristics of the readout coil and on the coupled SR sensor.

To characterize the response of the platform, we loaded a 500- $\mu$-diameter colon cancer spheroid in the gap of each SR and measured the resulting induced voltage shift $\Delta V$ (Figure $3 c$ ). Despite the identical SR dimensions, each sensing unit featured a different response upon addition of the microtissues due to the different quality factors of the resonators, so that the induced voltage variations ranged from $-0.59 \mathrm{mV}$ to $-0.15 \mathrm{mV}$. By normalizing the induced voltage variation to the quality factor of each sensing unit (i.e. by dividing the induced voltage shift by the corresponding $\mathrm{Q}$ factor), a homogeneous signal response could be obtained across the whole platform. The normalized voltage response upon addition of the test microtissues amounted to $\Delta V_{\text {norm }}=-11.2 \pm 0.3 \mu V$ (Figure 3c).

\section{Continuous Recording of Microtissue Spheroids}

To validate the capability of the platform to continuously monitor the growth of cancer microtissues, we loaded six HCT-116 spheroids of $300 \mu \mathrm{m}$ diameter in six different wells of the platform, and we measured the induced signal changes every 15 minutes during 4 days of culturing. The time interval between measurements enabled to detect any variation in spheroid size, growth rate, or tissue death, all of which have typical dynamics at the time scale of hours ${ }^{11}$.

The signal variation, obtained with a cancer spheroid, and representative microscopy images taken during culturing in the platform are shown in Figure 4a. Due to medium evaporation and medium exchange after 48 hours, the salt concentration in the culture well changed during the measurement. The increase of the salt concentration in the SR gap caused a decrease of the overall SR impedance and an increase of the capacitance of the SR gap, which induced a variation in the resonance frequency of the sensing unit and a concurrent increase in the voltage signal magnitude (see Supporting Information). In contrast, the 
presence and growth of a microtissue caused an increase of the overall SR impedance and a decrease in the voltage signal magnitude. Microtissue growth and medium evaporation hence induced opposite effects on the voltage signal magnitude, as, according to Eq. 2, the voltage magnitude at resonance is inversely proportional to the overall SR impedance. Therefore, we observed a non-monotonic variation of the voltage signal amplitude in Fig. 4a despite the continuous growth of the microtissue.

Upon medium exchange $(\mathrm{t}=48 \mathrm{~h})$, the original salt concentration was restored, and the resonance frequency of the sensing unit recovered to the initial value, while $\Delta V$ showed a discontinuity due to the sudden increase in the SR impedance. Variations in medium composition, such as increases in salt concentration, can potentially affect the status and viability of the cells during culturing. ${ }^{27}$ Although this effect can be reduced by operation in high-humidity environments, i.e., in a cell-culture incubator and with humidity pads in close proximity, medium evaporation during multi-day experiments cannot be completely avoided.

As the change in voltage signal magnitude upon evaporation was correlated to the observed variations in resonance frequency, information about the frequency change could be used to determine and compensate for the concurrent voltage-signal-magnitude change. Frequency variations are correlated to changes in the capacitance of the SR gap and, therefore, only depend on the dielectric properties of the medium, most prominently its salt concentration (more information in the Supporting Information). To decouple evaporation-related and spheroid-growth-related changes in the recorded voltage, we monitored the variation of the resonance frequency of the sensing unit during the multi-day assay. We first measured the relationship between variations of the resonance frequency and the corresponding induced changes of the voltage signal amplitude by having only medium in a culture well (without microtissue) and letting it evaporate (Figure $4 \mathrm{~b}$ ). The obtained frequency-voltage calibration curve was then used to compensate for the signal variations in the microtissue recordings caused by up-concentration of electrolytes as a consequence of medium evaporation. The use of the resonance frequency of the readout coil for detecting signal variations due to evaporation simplifies the experimental setup and allows for assessing the exact medium conditions in parallel to the growth measurements. This direct assessment is preferable to measuring medium properties by means of additional or external sensors. The evaporationcompensated voltage shift $\Delta V_{C}$ shows a monotonic and steady decrease throughout the whole recording, in accordance with the continuous growth of the cancer spheroid (Figure $4 c)$.

The capability of the system to monitor, in parallel, the size variation of multiple spheroids is shown in Figure 4d. The growth of six HCT-116 microtissues was monitored for 96 hours using the wireless impedance-based detection. Frequency-based evaporation compensation and signal normalization were applied to enable the comparison of the recordings acquired from SRs with different resonance frequencies and quality factors. The normalized signals ranged between $-3.8 \pm 0.1 \mu \mathrm{V}$ and $-13.4 \pm 0.7 \mu \mathrm{V}$, as the spheroids grew from $291.8 \pm 5.3 \mu \mathrm{m}$ diameter to $520.7 \pm 20.3 \mu \mathrm{m}$ during the recording, as can be seen from the microscopy characterization of the microtissues. The system, therefore, presented a normalized sensitivity of $-41.9 \pm 0.5 \mathrm{nV} / \mu \mathrm{m}$ (Figure $\mathrm{S} 6$ ). 


\section{Monitoring Drug Efficacy}

As an exemplary case study, we monitored during 96 hours the growth and growth inhibition of HCT-116 cancer spheroids under standard medium conditions (control samples) and of spheroids that were exposed to different concentrations of doxorubicin $(0.1 \mu \mathrm{M}, 1 \mu \mathrm{M}$, and $10 \mu \mathrm{M}$ ), a potent chemotherapeutic $\operatorname{drug}^{28}$ (Figure 5a). To validate our findings, the size of the spheroids was also characterized by microscopy imaging at day 0 , day 2 , and day 4 , and spheroid viability was measured via an end-point ATP assay.

The monotonous decrease of $\Delta V_{C, N o r m}$ of the control and 0.1- $\mu \mathrm{M}$-doxorubicin samples indicated that the cancer spheroids continuously grew under both conditions. The different slopes and signal amplitudes resulted from the difference in growth rates and final spheroid size, which was caused by exposure to the drug. The spheroids, exposed to higher doxorubicin concentrations, only showed a small size increase during the first $\sim 20$ hours of the assay, with a dose-dependent behavior. The signals of the 1- $\mu \mathrm{M}$ samples remained essentially constant after an initial growth phase, which indicated that the microtissue growth was arrested upon drug exposure. The signals recorded from the spheroids exposed to $10 \mu \mathrm{M}$ doxorubicin showed the trend to increase after a first brief tissue growth phase, and the signal amplitude finally exceeded that of the initial signals. These results indicated that, after inhibition of tissue growth, the spheroids started shrinking and decreased in size.

By using the system parameters, obtained during the platform validation, the normalized voltage shifts could be translated into size-variation information for the different tested conditions. Accordingly, the diameter of the microtissues under standard culture conditions was estimated to increase by more than $200 \mu \mathrm{m}$ during the four days of culturing, while the tissues exposed to $0.1 \mu \mathrm{M}$ doxorubicin featured a much less growth and a final size increase of only $\sim 100 \mu \mathrm{m}$ in diameter. The diameter of the microtissues exposed to $1 \mu \mathrm{M}$ drug concentrations only increased by $\sim 20 \mu \mathrm{m}$ during the first 24 hours of incubation, while their growth was then arrested. Microtissues exposed to $10 \mu \mathrm{M}$ doxorubicin were suppressed in growth from the beginning and shrank by $\sim 15 \mu \mathrm{m}$ in diameter.

The impedance recordings and the corresponding estimated size variations were in very good agreement with the sizes determined from microscopy images that were acquired throughout the assay. The microscopy data confirmed the growth, growth inhibition and shrinkage of the cancer microtissues upon exposure to the different drug concentrations (Figure 5b-c). The results of the ATP measurements, performed at the end of the assay, further confirmed the impedance-based measurements (Figure 5d).

\section{Conclusion}

The sensing system presented here enables electrical, wireless assessment of the dielectric characteristics of multiple microtissue spheroids in parallel. The detection of variations in the growth rate of cancer microtissue spheroids that were exposed to different concentrations of doxorubicin demonstrates the potential of the system for use in drug-screening application. The electrical measurements were validated by microscopy imaging and endpoint ATP quantification, both of which confirmed the observed dose-dependent effects on microtissue growth. The sensing system features a simple experimental setup, which 
includes an array of readout coils, split-ring sensors, and pyramidal well structures for tissue culturing and tissue placement. Owing to the small dimensions, the whole platform can easily fit into a standard cell-culture incubator and does not require modifications or specialized lab equipment.

Cable connections, which could interfere with the handling of the platform, are not required to interrogate the integrated sensors. Therefore, the presented approach could be easily integrated with lab-automation equipment, such as automatic liquid dispensers and robotic arms for increased throughput. The SR sensor dimensions were optimized to adhere to standard microtiter-plate dimensions, while avoiding cross talk between adjacent readout coils and sensor units. A higher number of sensing units can be integrated within a single platform to further increase throughput by reducing the SR and coil sizes and by tuning the resonance frequencies of the coils in the readout board. Although this approach would pose more stringent requirements on the board-to-chip distance and on the alignment due to the smaller sensor size, a sensor of $\sim 1 \mathrm{~mm}$ diameter would nevertheless retain a wirelesstransmission distance of $\sim 1 \mathrm{~mm}$, and it would be compatible with a well pitch of $4.5 \mathrm{~mm}$, which corresponds to the standard 384 well-plate format.

The continuous recording of two parameters, namely voltage amplitude and resonance frequency, was demonstrated to provide information on both, spheroid growth and medium conditions including the effects of evaporation. The available information was used to normalize the recorded signals during long-term culturing and to estimate signal variations caused by medium evaporation. The detection of resonance frequency variations can also be used for measuring fluctuations in the electrolyte concentration in the cell-culture medium and to monitor culture conditions during multi-day assays.

\section{Supplementary Material}

Refer to Web version on PubMed Central for supplementary material.

\section{Acknowledgment}

This work was supported by the National Natural Science Foundation of China (Grant No. 61801110) and the China Scholarship Council (File No. 201806095023), the Swiss Commission for Technology and Innovation (contract: 25727.1 PFLS-LS: "Broadband high-accuracy impedance analyzer") and by the Swiss National Science Foundation (contract CR32I2_166329: "Infected body-on-chip"). The authors would like to thank Julia Alicia Boos and Thi Phuong Oanh Nguyen, both at ETH Zurich, for help with the ATP assays.

\section{Abbreviations}

LC inductor capacitor

PCB printed circuit board

PDMS polydimethylsiloxane

SR $\quad$ split ring 


\section{References}

(1). Achilli TM, Meyer J, Morgan JR. Advances in the Formation, Use and Understanding of MultiCellular Spheroids. Expert Opin Biol Ther. 2012; 12(10):1347-1360. [PubMed: 22784238]

(2). Zanoni M, Piccinini F, Arienti C, Zamagni A, Santi S, Polico R, Bevilacqua A, Tesei A. 3D Tumor Spheroid Models for in Vitro Therapeutic Screening: A Systematic Approach to Enhance the Biological Relevance of Data Obtained. Sci Rep. 2016 Jan.6:1-11. [PubMed: 28442746]

(3). Esch MB, Smith AST, Prot JM, Oleaga C, Hickman JJ, Shuler ML. How Multi-Organ Microdevices Can Help Foster Drug Development. Adv Drug Deliv Rev. 2014; 69-70:158-169.

(4). Knight E, Przyborski S. Advances in 3D Cell Culture Technologies Enabling Tissue-like Structures to Be Created in Vitro. J Anat. 2015; 227:746-756. [PubMed: 25411113]

(5). Asthana A, Kisaalita WS. Microtissue Size and Hypoxia in HTS with 3D Cultures. Drug Discov Today. 2012; 17(15-16):810-817. [PubMed: 22484546]

(6). Friedrich J, Seidel C, Ebner R, Kunz-Schughart LA. Spheroid-Based Drug Screen: Considerations and Practical Approach. Nat Protoc. 2009; 4:309-324. [PubMed: 19214182]

(7). Langhans SA. Three-Dimensional in Vitro Cell Culture Models in Drug Discovery and Drug Repositioning. Front Pharmacol. 2018 Jan.9:6. [PubMed: 29410625]

(8). Sart S, Tomasi RFX, Amselem G, Baroud CN. Multiscale Cytometry and Regulation of 3D Cell Cultures on a Chip. Nat Commun. 2017; 8

(9). Boutin ME, Voss TC, Titus SA, Cruz-Gutierrez K, Michael S, Ferrer M. A High-Throughput Imaging and Nuclear Segmentation Analysis Protocol for Cleared 3D Culture Models. Sci Rep. 2018; 8

(10). Eichler M, Jahnke HG, Krinke D, Müller A, Schmidt S, Azendorf R, Robitzki AA. A Novel 96Well Multielectrode Array Based Impedimetric Monitoring Platform for Comparative Drug Efficacy Analysis on 2D and 3D Brain Tumor Cultures. Biosens Bioelectron. 2015; 67:582-589. [PubMed: 25445619]

(11). Poenick S, Jahnke HG, Eichler M, Frost S, Lilie H, Robitzki AA. Comparative Label-Free Monitoring of Immunotoxin Efficacy in 2D and 3D Mamma Carcinoma in Vitro Models by Impedance Spectroscopy. Biosens Bioelectron. 2014; 53:370-376. [PubMed: 24184957]

(12). Wang X, Cirit M, Wishnok JS, Griffith LG, Tannenbaum SR. Analysis of an Integrated Human Multiorgan Microphysiological System for Combined Tolcapone Metabolism and Brain Metabolomics. Anal Chem. 2019; 91:8667-8675. [PubMed: 31181164]

(13). Chawla K, Modena MM, Ravaynia PS, Lombardo FC, Leonhardt M, Panic G, Bürgel SC, Keiser J, Hierlemann A. Impedance-Based Microfluidic Assay for Automated Antischistosomal Drug Screening. ACS Sensors. 2018; 3:2613-2620. [PubMed: 30426744]

(14). Wu H, Yang Y, Bagnaninchi PO, Jia J. Electrical Impedance Tomography for Real-Time and Label-Free Cellular Viability Assays of 3D Tumour Spheroids. Analyst. 2018; 143(17):4189_ 4198. [PubMed: 30070264]

(15). Sun T, Morgan H. Single-Cell Microfluidic Impedance Cytometry: A Review. Microfluid Nanofluidics. 2010; 8(4):423-443.

(16). Kloß D, Kurz R, Jahnke HG, Fischer M, Rothermel A, Anderegg U, Simon JC, Robitzki AA. Microcavity Array (MCA)-Based Biosensor Chip for Functional Drug Screening of 3D Tissue Models. Biosens Bioelectron. 2008; 23:1473-1480. [PubMed: 18289841]

(17). Bürgel SC, Diener L, Frey O, Kim JY, Hierlemann A. Automated, Multiplexed Electrical Impedance Spectroscopy Platform for Continuous Monitoring of Microtissue Spheroids. Anal Chem. 2016; 88:10876-10883. [PubMed: 27650426]

(18). Schmid YRF, Bürgel SC, Misun PM, Hierlemann A, Frey O. Electrical Impedance Spectroscopy for Microtissue Spheroid Analysis in Hanging-Drop Networks. ACS Sensors. 2016; 1:10281035.

(19). Huang QA, Dong L, Wang LF. LC Passive Wireless Sensors Toward a Wireless Sensing Platform: Status, Prospects, and Challenges. J Microelectromechanical Syst. 2016; 25(5):822841. 
(20). Mannoor MS, Tao H, Clayton JD, Sengupta A, Kaplan DL, Naik RR, Verma N, Omenetto FG, McAlpine MC. Graphene-Based Wireless Bacteria Detection on Tooth Enamel. Nat Commun. 2012; 3

(21). Chen LY, Tee BCK, Chortos AL, Schwartz G, Tse VJ, Lipomi D, Wong HSP, McConnell MV, Bao Z. Continuous Wireless Pressure Monitoring and Mapping with Ultra-Small Passive Sensors for Health Monitoring and Critical Care. Nat Commun. 2014; 5

(22). Song SH, Park JH, Chitnis G, Siegel RA, Ziaie B. A Wireless Chemical Sensor Featuring Iron Oxidenanoparticle-Embedded Hydrogels. Sensors Actuators, B Chem. 2014; 193:925-930.

(23). Economou EN, Koschny T, Soukoulis CM. Strong Diamagnetic Response in Split-RingResonator Metamaterials: Numerical Study and Two-Loop Model. Phys Rev B - Condens Matter Mater Phys. 2008; 77

(24). Benson K, Cramer S, Galla HJ. Impedance-Based Cell Monitoring: Barrier Properties and Beyond. Fluids Barriers CNS. 2013; 10:5. [PubMed: 23305242]

(25). Gawad S, Cheung K, Seger U, Bertsch A, Renaud P. Dielectric Spectroscopy in a Micromachined Flow Cytometer: Theoretical and Practical Considerations. Lab Chip. 2004; 4:241-251. [PubMed: 15159786]

(26). Bürgel SC, Escobedo C, Haandbæk N, Hierlemann A. On-Chip Electroporation and Impedance Spectroscopy of Single-Cells. Sensors Actuators, B Chem. 2015; 210:82-90.

(27). Gilbert DF, Mofrad SA, Friedrich O, Wiest J. Proliferation Characteristics of Cells Cultured under Periodic versus Static Conditions. Cytotechnology. 2019; 71:443-452. [PubMed: 30515656]

(28). Lüpertz R, Wätjen W, Kahl R, Chovolou Y. Dose- and Time-Dependent Effects of Doxorubicin on Cytotoxicity, Cell Cycle and Apoptotic Cell Death in Human Colon Cancer Cells. Toxicology. 2010; 271:115-121. [PubMed: 20346999] 


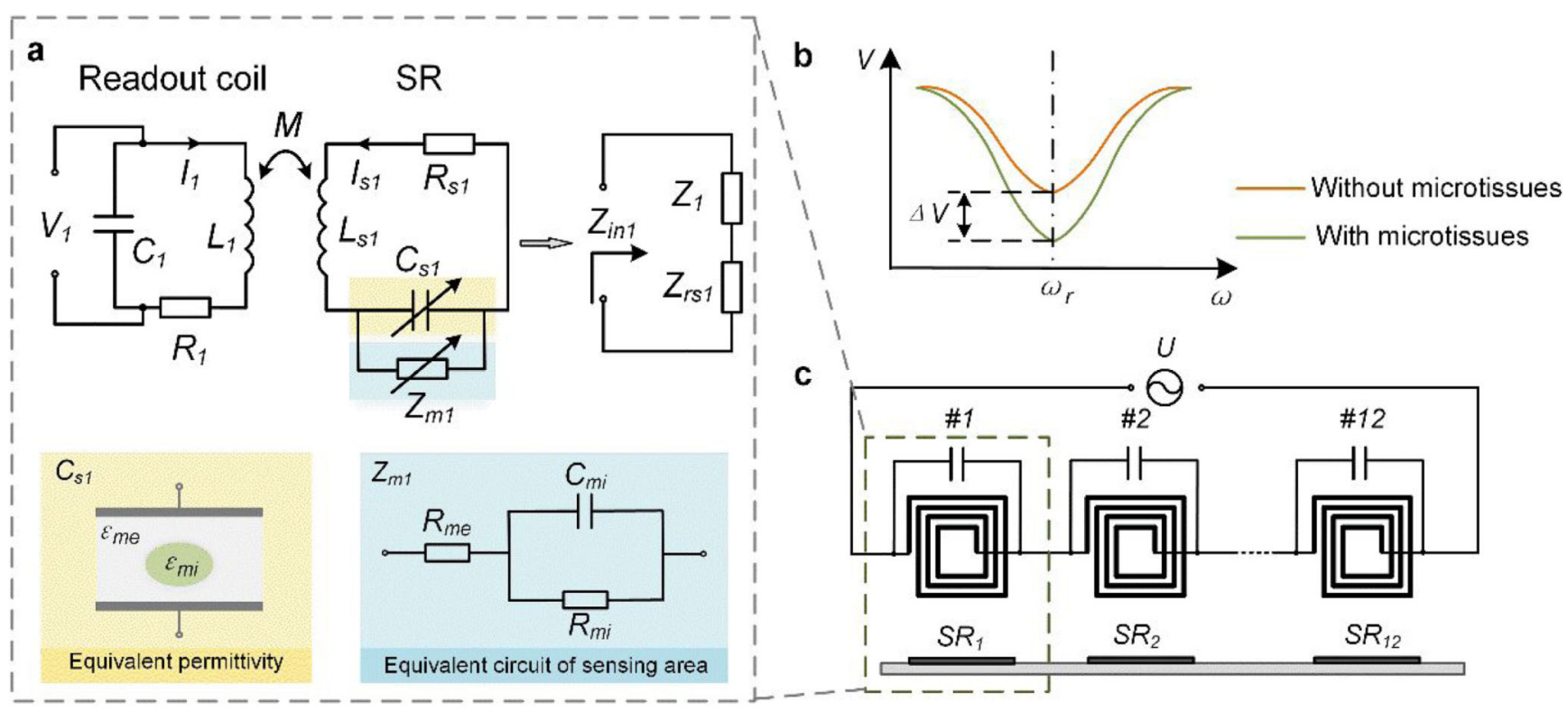

Figure 1.

Electrical equivalent model of the SR sensing unit. (a) The electrical equivalent circuit of a wireless sensing unit includes the readout coil, with its intrinsic parasitic capacitance and resistance, and the SR equivalent circuit. (b) The potential drop across the readout circuit features a minimum at the resonance frequency $\omega_{\mathrm{r}}$. (c) The schematic depicts a parallelized sensing system with multiple readout coils in series, which are paired with the corresponding SR sensors. 


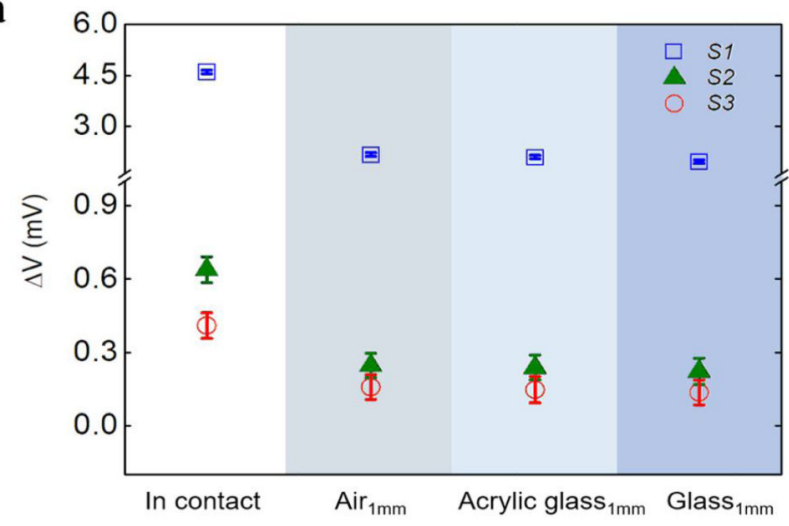

b

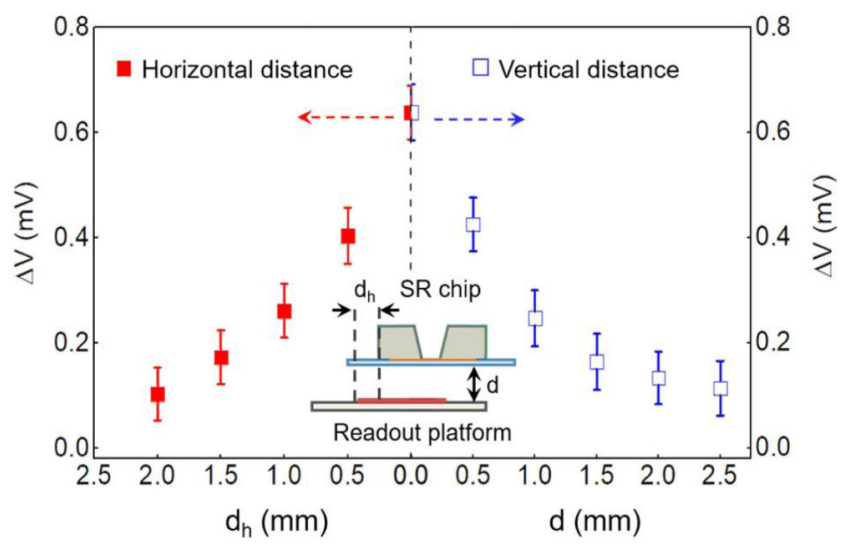

Figure 2.

(a) Three sensor samples $S 1\left(r_{S O}=5 \mathrm{~mm}, w=1 \mathrm{~mm}\right), S 2\left(r_{S O}=2 \mathrm{~mm}, w=1 \mathrm{~mm}\right)$, and $S 3\left(r_{S o}=2\right.$ $\mathrm{mm}, w=0.5 \mathrm{~mm}$ ) were tested under various conditions. The measurements in the white panel were measured with the sensors being in physical contact with the readout board; the grey, light blue and dark blue panels show the results acquired upon introducing different dielectric materials, namely air, acrylic glass and glass, of $1 \mathrm{~mm}$ thickness between the sensors and the readout platform. The error bars indicate the standard deviation $(n=3)$. (b) Using sensor S2 we increased the vertical (right panel) and horizontal distance (left panel) between the readout board and the sensor. The average induced $\Delta \mathrm{V}$ were recorded after loading a HCT-116 spheroid of $500 \mu \mathrm{m}$ diameter. The error bars indicate the standard deviation $(n=3)$. 


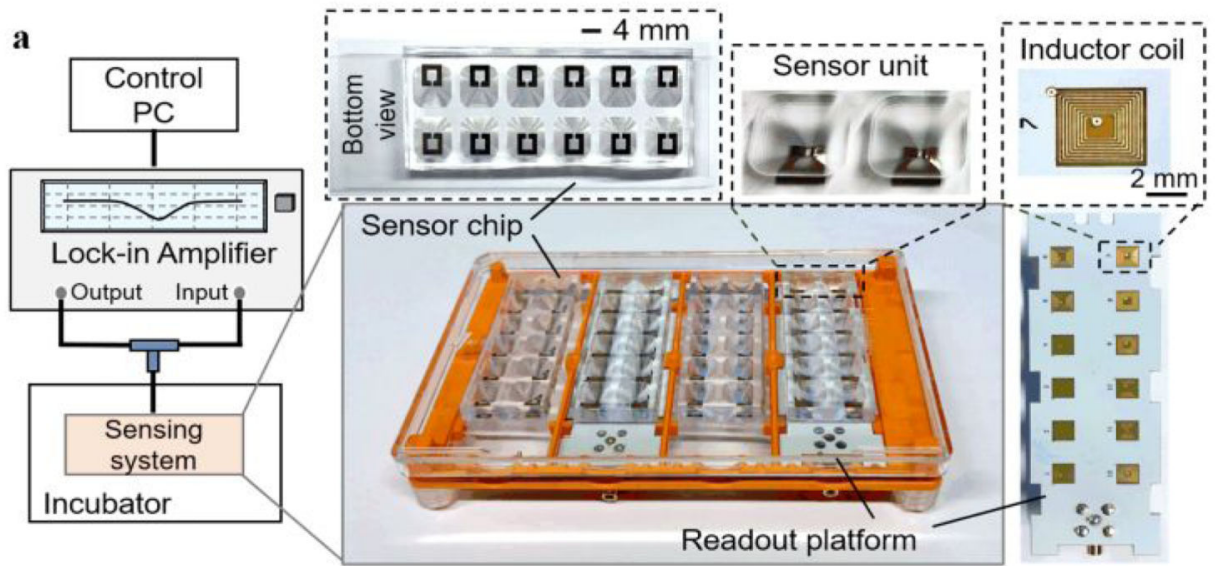

b

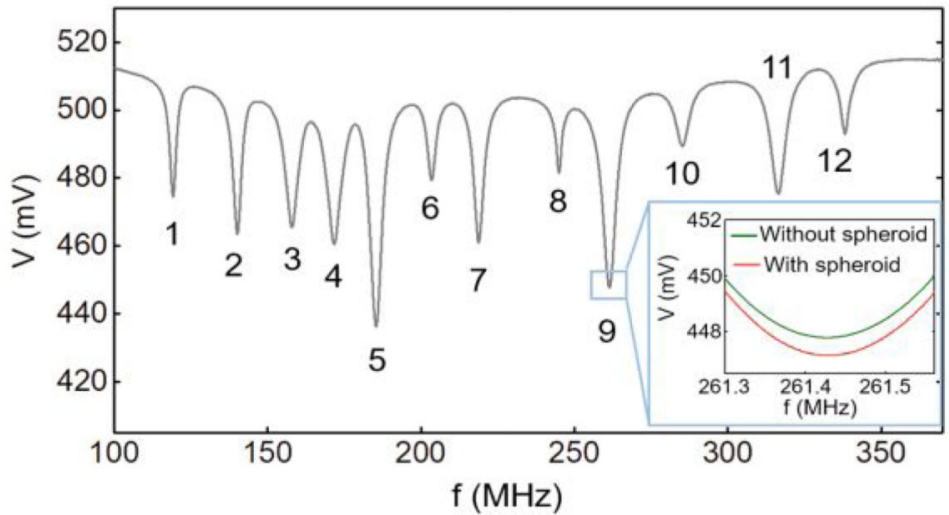

c

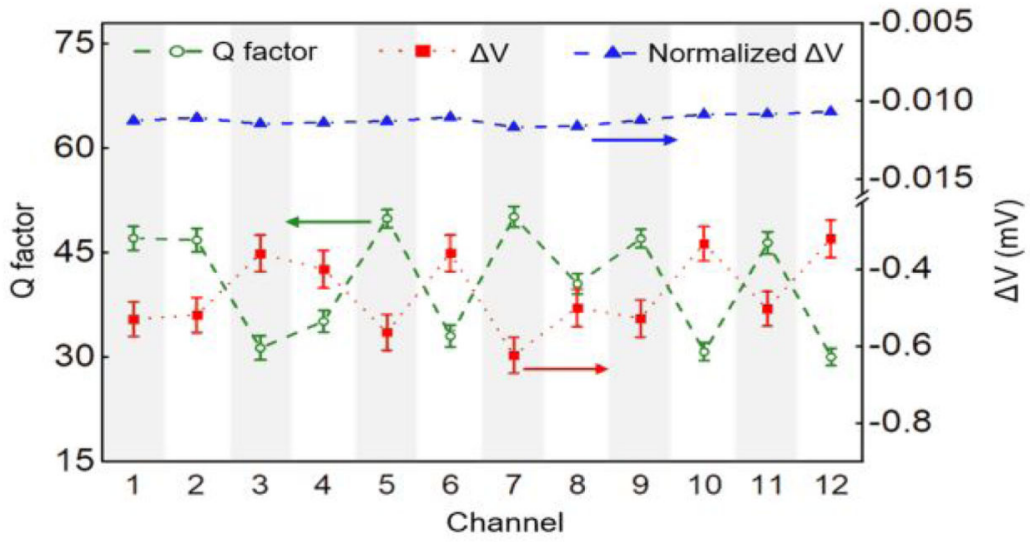

Figure 3.

Parallel sensing platform. (a) Measurement setup. The readout PCBs and the SR sensor chips were affixed on a microscopy-slide holder. (b) A frequency sweep from $100 \mathrm{MHz}$ to $370 \mathrm{MHz}$. The twelve resonance peaks of the readout platform are clearly visible. The inset shows a representative voltage shift at resonance induced by a $500-\mu \mathrm{m}$-diameter spheroid in the SR gap. (c) The quality factors $Q$ of the 12 sensing units in the platform and the respective voltage shifts $\Delta \mathrm{V}$ induced by the $500-\mu \mathrm{m}$ sphe-roid in the SR gap. The presence of the microtissue only affects the signal amplitude but not the resonance frequency of the 
sensing unit. (c) The quality factors $Q$ of the 12 sensing units in the platform and the respective voltage shifts $\Delta \mathrm{V}$ induced by the $500-\mu \mathrm{m}$ spheroid in the SR gap. The bars indicate one standard deviation $(n=4)$. By dividing the response of each sensing unit by the resonator quality factor, a uniform response was obtained from the twelve sensing units, which is denoted "Normalized $\Delta \mathrm{V}$ ". 

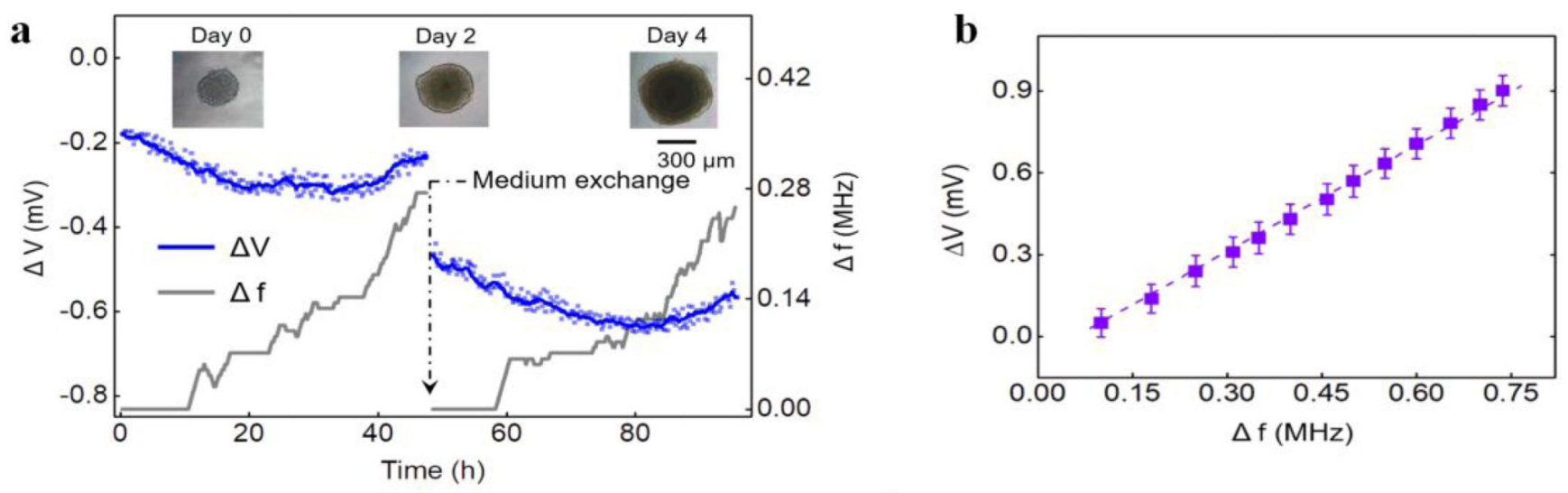

c
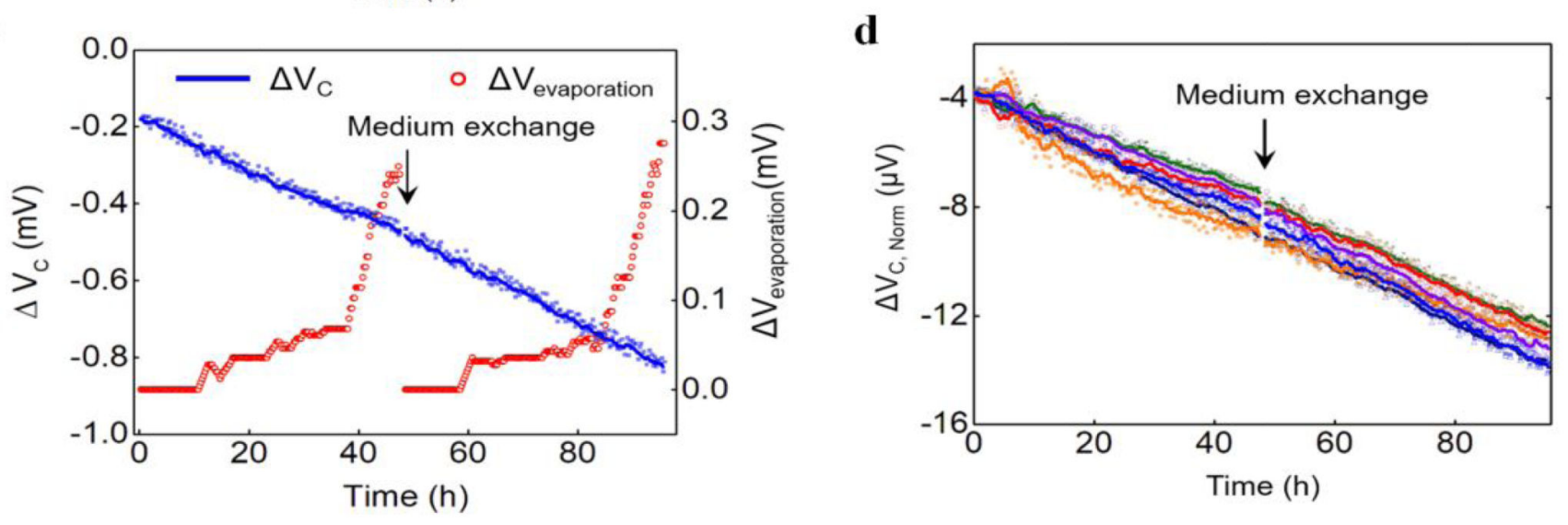

Figure 4.

Continuous recording of microtissue growth and evaporation estimation method. (a)

Variations in voltage signal amplitude (in blue) and resonance frequency (in grey) of a growing microtissue spheroid during 90 hours of culturing. A clear signal discontinuity is visible after 48 hours of culturing due to medium exchange. The insets show microscopy images of the microtissue acquired on day 0 , day 2 , and day 4 , respectively. (b) Correlation between resonance frequency shift and voltage change, caused by evaporation, which was used as a basis for compensation of signal variations through frequency monitoring. The error bars indicate the standard deviation $(n=6)$. (c) In blue, the results of (a) after frequencysignal compensation. The red points show the voltage-signal amplitude variations caused by medium evaporation. The evaporation-induced voltage amplitude changes were estimated from the resonance frequency variations by using the calibration curve reported in (b). (d) Frequency-compensated signal variations $\Delta \mathrm{V}_{\mathrm{C}}$, Norm of six cancer spheroids measured in parallel. Frequency compensation as in (c) was used to remove the artifacts induced by medium evaporation using the calibration in (b). 
a

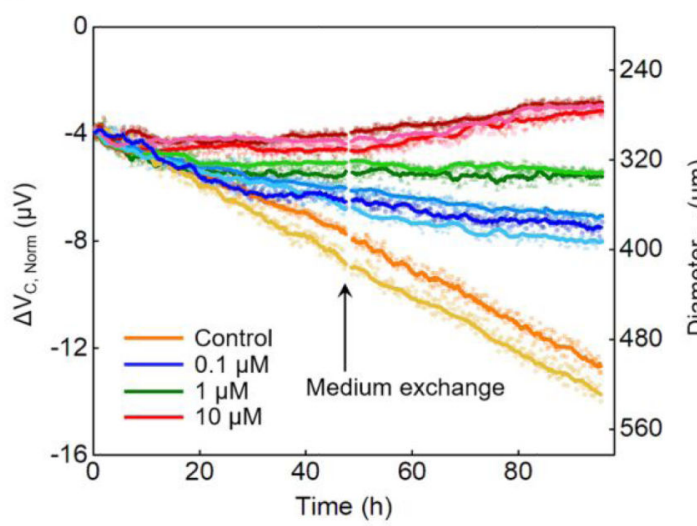

b

$$
\text { b }
$$

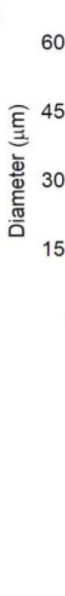

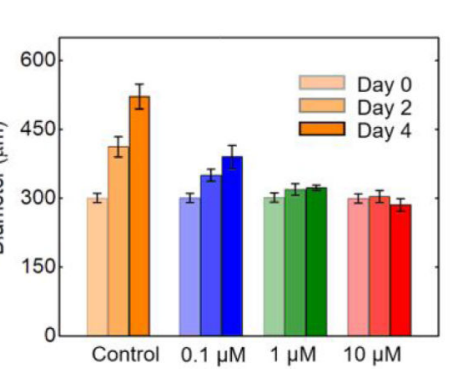

Control $\quad 0.1 \mu \mathrm{M} \quad 1 \mu \mathrm{M} \quad 10 \mu \mathrm{M}$

c

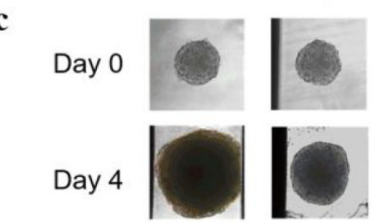

d

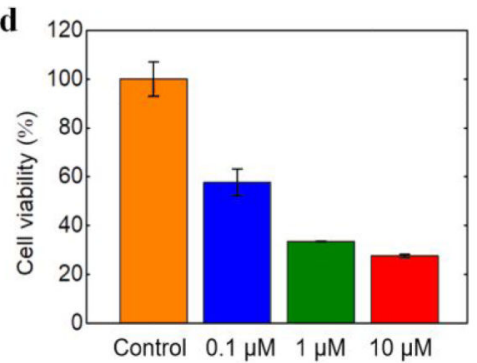

Control $0.1 \mu \mathrm{M} \quad 1 \mu \mathrm{M} \quad 10 \mu \mathrm{M}$

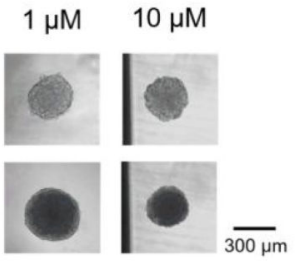

Figure 5.

Effect of doxorubicin on HCT-116 spheroids. (a) Normalized and frequency-compensated $\Delta \mathrm{V}_{\mathrm{C}, \text { Norm }}$ values of cancer spheroids exposed to $0.1,1,10 \mu \mathrm{M}$ doxorubicin, and to standard medium conditions. The medium was exchanged after 48 hours. By using the previously calculated sensor sensitivity, the normalized voltage signals were converted into spheroid diameters. (b) The average diameter of the spheroids measured with a plate scanner at day 0 , 2 and 4 of incubation. The error bars represent the mean absolute errors $(n=2)$. (c)

Representative microscopy images of the spheroids at day 0 and day 4. (d) ATP measurements of the cancer spheroids after 96 hours of culturing in the platform. The values were normalized to the ATP value obtained from the control samples. The error bars indicate the mean absolute errors $(n=2)$. 